\title{
The influence of the interplanetary medium on SuperDARN radar scattering occurrence
}

\author{
P. Ballatore ${ }^{1}$, J. P. Villain ${ }^{1}$, N. Vilmer $^{2}$, M. Pick $^{2}$ \\ ${ }^{1}$ LPCE/CNRS, Av. de la Recherche Scientifique, Orleans, 45071 - France \\ 2 DASOP, UMR 8645 CNRS, Observatoire de Meudon, Meudon, 92195 - France
}

Received: 3 May 2000 / Revised: 4 October 2000 / Accepted: 6 October 2000

\begin{abstract}
The effects of the characteristics of the interplanetary medium on the radar scattering occurrence, related to the whole array of SuperDARN radars installed in the Northern Hemisphere, have been studied over a two-year period. Statistically significant correlations of the variation of the scattering occurrence are found with the merging electric field and with the negative $B_{z}$ component of the interplanetary magnetic field, independent of the seasonal period considered. This result demonstrates that the merging rate (and in particular the reconnection process) between the interplanetary magnetic field and the magnetosphere is a relevant factor affecting the occurrence of scattering. For comparison, we note that no statistically significant correlations are obtained when the interplanetary ion density or the solar wind speed are considered, although also these variables affect to a small degree the scattering occurrence variation. The study of the latitudinal and magnetic local time dependence of the observations shows an association between the considered correlation and the location of the auroral oval and the cusp/cleft region.
\end{abstract}

Key words: Ionosphere (ionospheric irregularities) Magnetospheric physics (solar wind-magnetosphere interactions) - Radio science (ionospheric physics)

\section{Introduction}

As a part of the SuperDARN (Super Dual Auroral Radar Network) initiative, six coherent-scatter HF (high frequency) radars are currently operating in the Northern Hemisphere and continuously monitoring the highlatitude ionosphere in the frequency range $8-20 \mathrm{MHz}$ (Greenwald et al., 1995). The level of scatter measured by the radars is highly variable depending on the global effects of the high-latitude phenomena.
The backscatter (or echoes) of the radar sounding signal radio wave, $k_{r}$, is generated by the presence of ionospheric irregularities with wave vectors, $k$, which correspond to the Bragg condition $k$ equal to $\pm 2 k_{r}$. It is known that the phase front of irregularities are aligned with the geomagnetic field lines $(k \perp B$, where $B$ is the magnetic field), so that echoes are generated if the $k_{r}$ vector of the sounding wave lies in the plane perpendicular to the local magnetic field. At HF frequencies the ionosphere can refract the $k_{r}$ vector so that it becomes orthogonal to the local magnetic field at $\mathrm{E}$ and $\mathrm{F}$ region altitudes (e.g., Villain et al., 1984; Milan et al., 1997). The occurrence of HF backscatter thus depends on both the presence of irregularities and the existence of a suitable HF propagation condition (e.g., Ruohoniemi et al., 1987; Ruohoniemi and Greenwald, 1997).

Previous studies on the rate of the scattering occurrence were reported by Ruohoniemi and Greenwald (1997) for the SuperDARN radar located at Goose Bay (Canada), and by Milan et al. (1997) for the CUTLASS radars. In both studies a significant dependence of the echo occurrence on magnetic local time (MLT) is found. Moreover, the diurnal variation of this occurrence is found to be significantly influenced by the season and by the geomagnetic activity (as indicated by the $K p$ index).

The present work is related to the effects of interplanetary parameters on the rate of scattering occurrence obtained when considering the array of all the six Northern Hemisphere SuperDARN radars in operation during the years 1997 and 1998.

\section{Data analysis}

\subsection{Measurements of SuperDARN scattering occurrence}

The fields-of-view of the SuperDARN radars in the Northern Hemisphere are shown in Fig. 1. Each radar carries out an azimuthal sweep through discrete beams pointing in 16 directions. In the common mode the backscatter is recorded in $45 \mathrm{~km}$ range steps from $180 \mathrm{~km}$ to nearly $3500 \mathrm{~km}$ from each radar. The scans are synchronized to begin on 2-min boundaries starting 


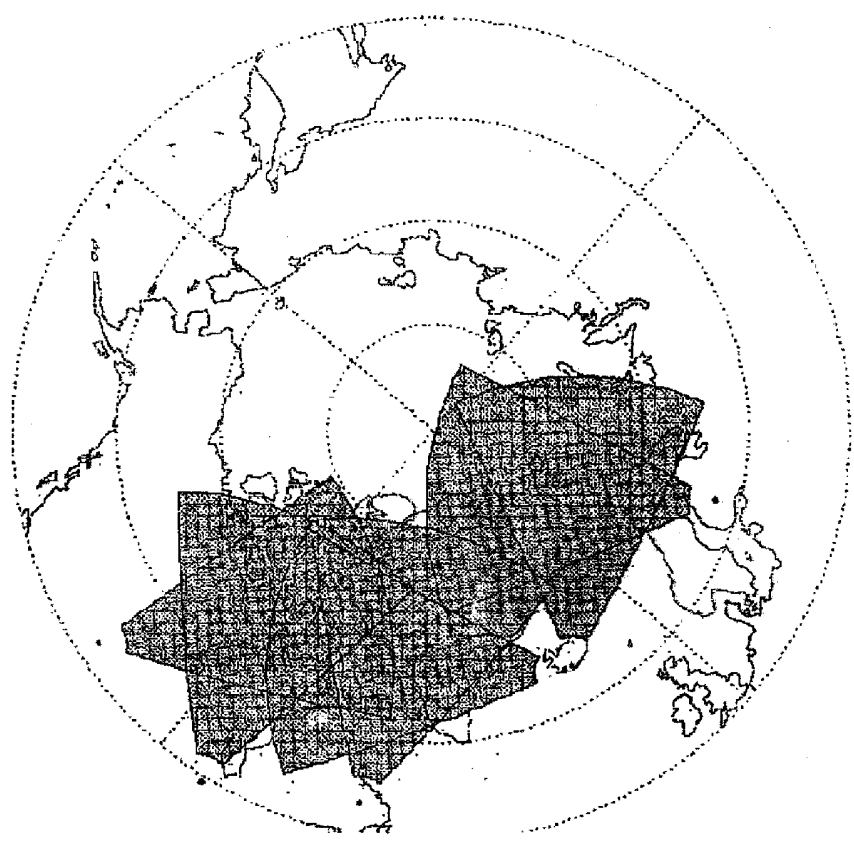

Fig. 1. Fields-of-view of the Northern Hemisphere SuperDARN radars available for the period of study considered

with 00:00 UT (Universal Time). For each scan the radars record the backscatter parameters including, in particular, power, noise and a flag indicating the presence of ground scatter. Ground scatter is distinguished by a near-zero doppler velocity and a low spectral width (e.g., Milan et al., 1997; Ruohoniemi and Greenwald, 1997) and it has been rejected in the data analysis presented here.

All the data available from all six radars operating in the Northern Hemisphere at the time of this study were considered. For the measurements of all radars considered together, the ratio, $R$, of the number of echoes with a signal-to-noise $(S / N)$ above $6 \mathrm{~dB}, N_{s}$, versus the total number of soundings, $N_{t}$, was calculated each 6-min UT intervals (i.e., three times the temporal resolution of the scanning). We note that the results presented in the following are not affected by a relatively small change of the $S / N$ level chosen as threshold for significant measurements, which can be considered arbitrary. However the level $6 \mathrm{~dB}$ in particular was selected to be higher than in previous studies of scattering occurrence $(S / N$ equal to $3 \mathrm{~dB}$ was chosen as sufficiently resolved backscatter by Ruohoniemi and Greenwald, 1997), in order to sort relatively stronger signals, possibly more independent of instrumental/noise effects. The parameter, $R$, is considered representative of the rate of the scattering occurrence.

$R=\Sigma_{\text {radars }} \Sigma_{\text {beams }} \Sigma_{\text {cells }}(N s) / \Sigma_{\text {radars }} \Sigma_{\text {beams }} \Sigma_{\text {cells }}(N t)$

We have considered the variation of $R, \delta R$, obtained after the subtraction of the average daily modulation calculated over one-month intervals. In the following we consider $\delta R$ instead of $R$ itself in order to exclude the effects due to the fact that the radars do not cover uniformely all longitude locations and that they rotate during the 24 hours of the day. $\delta R(U T)=R(U T)-\langle R\rangle(U T)$

Where $\langle R\rangle$ is the average of $R$ over a one-month period, calculated for each 6-min UT interval of the 24 daily hours. $R$ and $\langle R\rangle$ can be calculated separately for cells located at different magnetic latitude ranges. Thus we specify the CGM (corrected geomagnetic) latitude of $R$ and $\delta R$ (see Sect. 2.3). Moreover, by taking into account only radar cells located at specific MLT locations, $R$ and $\delta R$ can be computed for specific MLT ranges (see Sect. 2.4).

\subsection{Observations of the interplanetary medium with the WIND satellite}

The parameters of the interplanetary medium considered here are measured by the WIND satellite and they are: (a) solar wind proton density $(N)$; (b) solar wind speed $\left(V_{s w}\right)$; (c) interplanetary magnetic field (IMF).

Using the WIND measurements of the IMF and of the $V_{s w}$, we have calculated the merging electric field, $E_{\text {merg. }}$.

$E_{\text {merg }}=V_{s w} * B_{t} * \sin ^{2}(\phi / 2)$

Where $B_{t}$ is the projection of the IMF on the $Y-Z$ plane, in the considered GSM (geocentric solar magnetospheric) system of coordinates where the origin is in the center of the Earth, with $X$ pointing toward the Sun and $Y$ perpendicular to the Earth's dipole axis. The parameter $\phi$ in Eq. 3 (e.g., Kan and Lee, 1979) is the IMF clock angle between $B_{t}$ and the GSM $Z$-axis. In the Eq. 3, we note that the factor associated with $\phi$ is progressively increasing in the interval $0-1$ while $|\phi|$ increases between $0^{\circ}$ (corresponding to $B_{y}=0$ and positive $B_{z}$, i.e. northward IMF) and $180^{\circ}$ (corresponding to $B_{y}=0$ and negative $B_{z}$, i.e. southward IMF).

In Fig. 2 we show an example of the WIND observations. These parameters are illustrated together with $\delta R$ for the latitude range $60-67^{\circ} \mathrm{CGM}$ (bottom panel). The vertical dashed lines indicate the time interval of an interplanetary coronal mass ejection event. A clear relationship between $E_{\text {merg }}$ and the increase of $\delta R$ (similar to the results in Ballatore et al., 1999 ) is shown. No clear association is shown between $\delta R$ and $V_{s w}$ or $N$. We note that the increase of $\delta R$ is delayed by about two hours with respect to the increase of $E_{\text {merg }}$ and negative $B_{z}$. With the inclusion of this delay, and considering the interval included between the two vertical dashed lines, the correlation coefficient between $\delta R$ and IMF $B_{z}$ is -0.60 and between $\delta R$ and $E_{\text {merg }}$ it is 0.68 .

\subsection{Correlations between the HF scattering occurrence at different magnetic latitudes and the interplanetary parameters}

The time interval considered in the present study is restricted to the years 1997 and 1998 owing to WIND data availability (since 1997) and the fact that yearly sets 


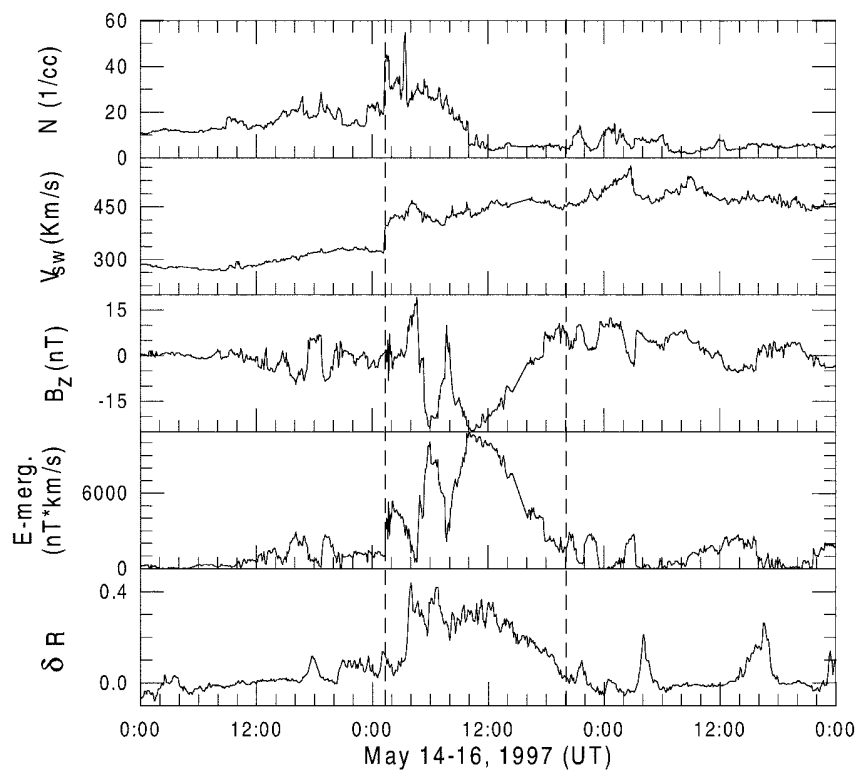

Fig. 2. Time series of interplanetary proton density $N$, solar wind speed $V_{s w}$, IMF $B_{z}$ component, the merging electric field $E_{\text {merg }}$, and the variation of the rate of scattering occurrence for the latitude $60-67^{\circ}$ CGM; the vertical dotted lines indicate the interplanetary CME event on May 15, 1997

of SuperDARN data were available until 1998 during the period of this study. We report here the results of an analysis considering the two years 1997-1998 together. No significant differences are found by considering each year separately.

For the period in question the correlation coefficients, $\rho$, between $\delta R$ and the different parameters of the interplanetary medium were calculated. If we calculate the probability that two uncorrelated variables have correlation coefficients equal to the ones found for negative IMF $B_{z}$ and for $E_{m e r g}$, with the same number of data points, we found that this probability is less than 0.001 , so that IMF $B_{z}$ and $E_{\text {merg }}$ can be considered to be correlated with a confidence greater than $99.9 \%$. In contrast, no significant correlation coefficients (of the order of 0.2 or smaller) were obtained for $N$ or $V_{s w}$.

We calculated the correlation coefficients $\rho$ separately for the different seasons: winter (January, February, November and December of 1997 and 1998), equinox (March, April, September, October of 1997 and 1998), and summer (May, June, July and August of 1997 and 1998) and for different values of CGM latitudes.

If we consider data average on 30-min averages and shift the $\delta R$ time series with respect to the time-serie $E_{\text {merg }}$ (or IMF $B_{z}$ ), we found that the correlation coefficients can be optimized with a time-shift of 60 min during the summer and equinox periods, and 90 min during the winter. In previous studies, a similar optimisation of correlations, obtained by shifting ground data with respect to satellite data, was interpreted in terms of the delay of ground effects due to the propagation of the solar wind from the satellite location to the magnetopause, and to the time necessary to the magnetospheric/ionospheric system to react by modify- ing its parameters in response to the changing of the interplanetary condition (e.g., Troshichev et al., 1988; Arnoldy, 1971). We can interpret the time-shift which optimizes our correlations in the same way, as average value of the delay of the response of scattering occurrence to interplanetary medium. We note, however, that the exact delay at each time is strongly dependent on the location of the satellite, the solar wind speed, the location of the magnetopause (which dynamically changes with interplanetary conditions, as described in Russell et al., 2000), the state of the ionosphere (in particular the ionization level, which changes with season) and geomagnetic activity. We recall that previous studies demonstrated that several interplanetary events, although having rather similar temporal profiles of the interplanetary parameters, show major differences in the magnetospheric response (e.g., Farrugia et al., 1998). In addition we note that in Fig. 2 (during the summer event shown) the $\delta R$ has a sharp increase around 2 UT hours later than the shock in the WIND data, not the estimated average $60 \mathrm{~min}$ value. The propagation time from the satellite to the magnetopause can be estimated at around $30 \mathrm{~min}$ at this time, so that the remaining $90 \mathrm{~min}$ is the time necessary for the $\delta R$ parameter to respond to the interplanetary configuration and this can be attributed to internal magnetospheric/ionospheric effects.

In the following we consider the optimized correlations with a time-shift for $\delta R$ (with respect to WIND data) of 60 min for equinox and summer and 90 min for winter. We note that the difference of the correlation coefficients for a shift slightly higher or smaller than $60 \mathrm{~min}$ (for summer or equinox) and $90 \mathrm{~min}$ (for winter) is of the order of $0.1-0.2$, therefore the best shift considered is not uniquely identified, due to the small difference associated with larger or smaller shifts.

We can further optimize the correlation coefficients by changing the amplitude of the latitude range considered for $\delta R$ : we find that the best correlation can be obtained for intervals of $7^{\circ}$. The correlation coefficients obtained with $7^{\circ}$ latitudinal ranges are higher than those obtained for different ranges, with differences of the order of 0.1 , independent of the season.

Considering the interplanetary/ground-based time shifts described above, we show in Fig. 3 the correlation coefficients, $\rho$, of $E_{m e r q}$ and IMF $B_{z}$ with $\delta R$ calculated over latitude ranges of 7 CGM degrees (the center of the 7-degree latitudinal interval, for each $\delta R$ considered, is indicated on the abscissa axes). The data points considered here are 30-min averages of $\delta R$, negative IMF $B_{z}$ and $E_{\text {merg }}$ derived as described in Sects. 2.1 and 2.2. The best correlation is obtained for the lowest latitudes (closer to the radars site as shown in Fig. 1). Moreover, by shifting the latitude range poleward the correlation between the interplanetary parameters and the occurrence of radar echoes decreases markedly, and there is no significant correlation above around $67^{\circ} \mathrm{CGM}$ and in particular for the latitudes not shown above $79^{\circ}$ CGM.

The decrease in correlation between $\delta R$ and $E_{\text {merg }}$ or negative $B_{z}$ for the highest latitudes, shown in Fig. 3, 
can be associated in part with the decrease in the number of echoes $R$. In Fig. 4 we show that the average of $R$, calculated for each seasonal interval over different latitude ranges. We note that $R$ in summer decreases at higher latitude, whereas in winter it remains equal to about 0.09 up to around $80^{\circ} \mathrm{CGM}$.

Figure 5 shows the scatter (for different seasons) between $\delta R$ and the interplanetary parameters negative IMF $B_{z}$ (Fig. 5a) and $E_{\text {merg }}$ (Fig. 5b). In this case $\delta R$ is computed in the latitude range $60-67^{\circ} \mathrm{CGM}$, where $\rho$ is maximum, and considering the 30 -min averages for the data. The correlations shown in Fig. 5a, b are significantly correlated at a confidence level above $99.9 \%$.

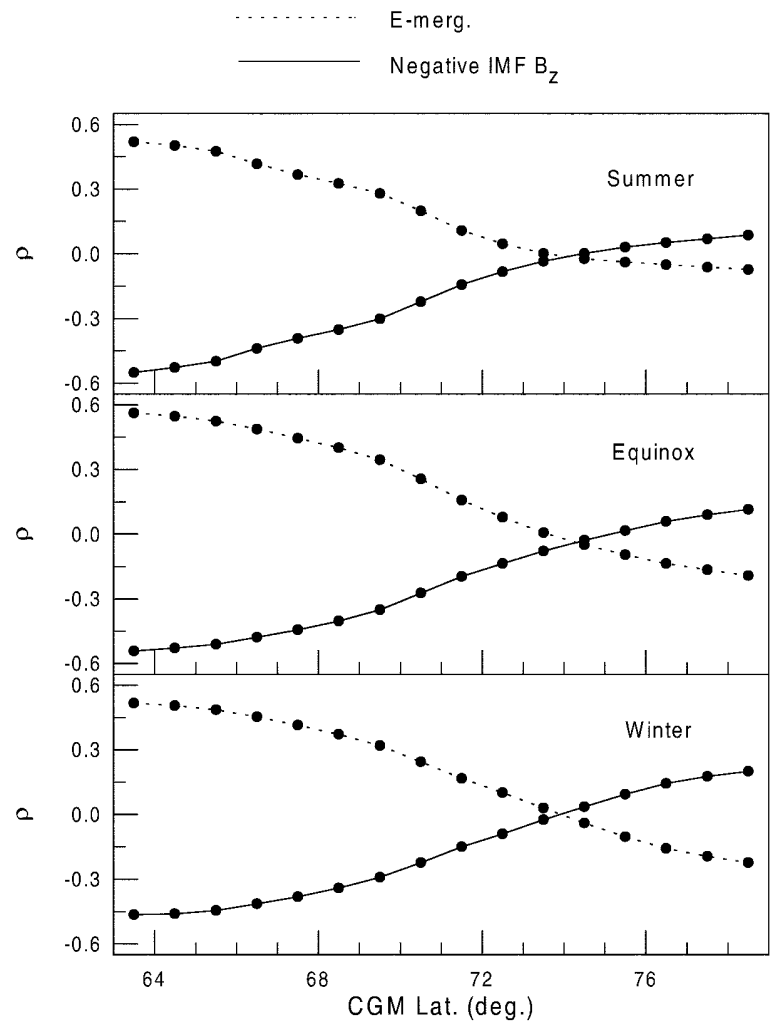

Fig. 3. Coefficients $\rho$ of the correlations between negative IMF $B_{z}$ or $E_{\text {merg }}$ and the variation of scattering occurrence, calculated over 7-degree latitude ranges centered at the indicated CGM latitude

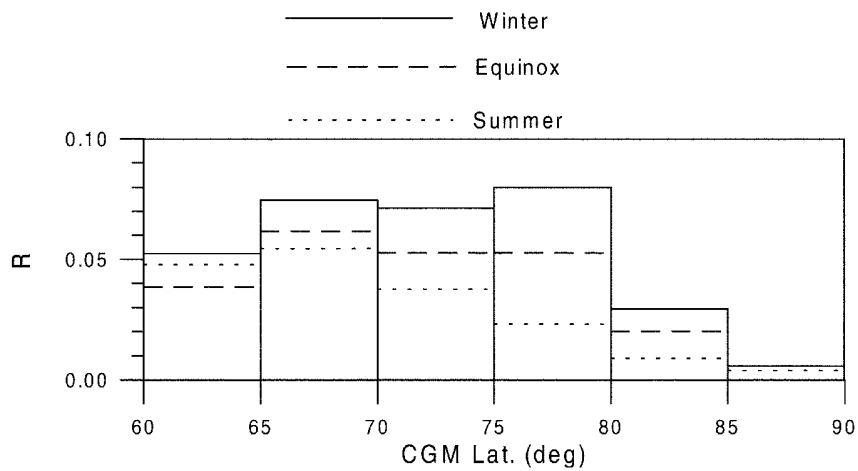

Fig. 4. Average of the scattering occurrence at different latitude ranges and for different seasons during 1997 and 1998
2.4 Correlations between the HF scattering occurrence at different MLT and the interplanetary parameters

In this section we show $\delta R$ for different ranges of MLT. After averaging the data on 30-min intervals, we
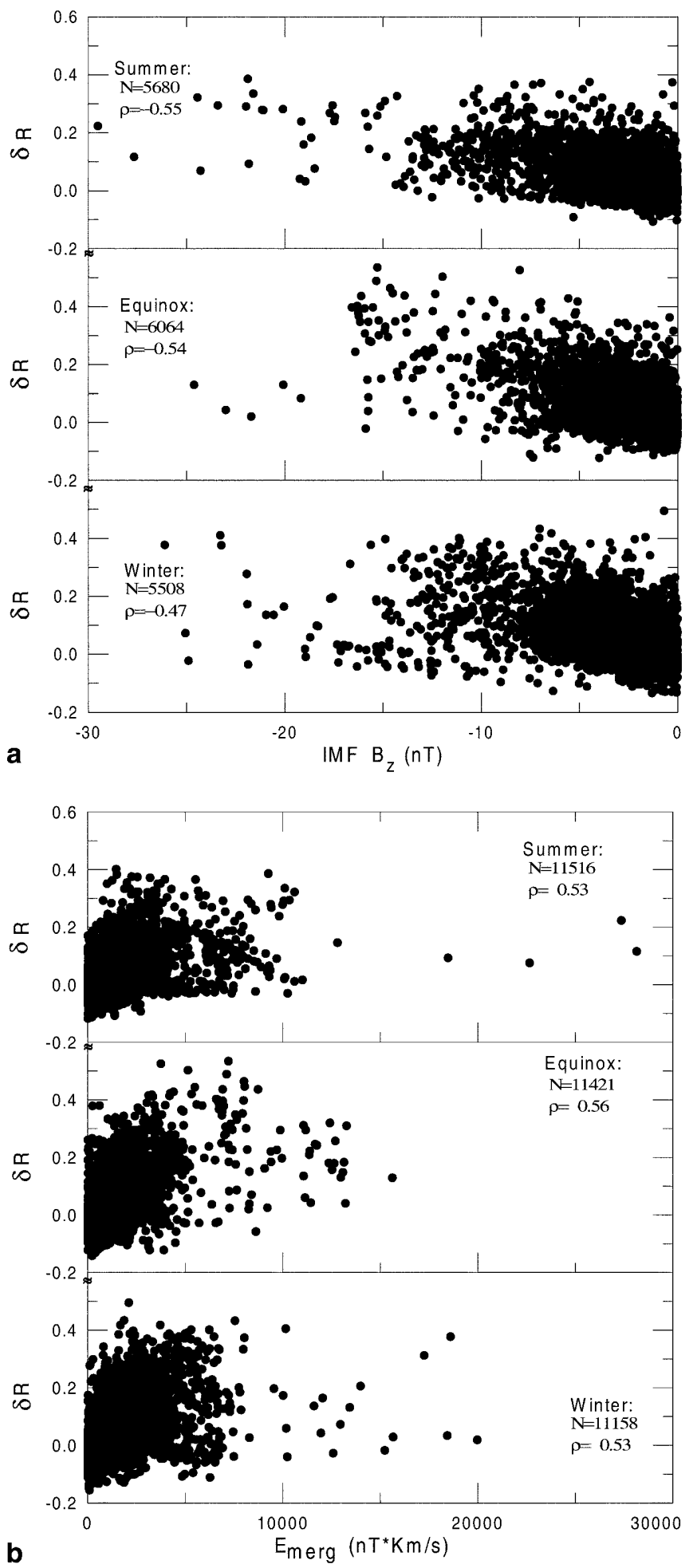

Fig. 5a, b. Variation of the scattering occurrence versus a negative IMF $B_{z}$, and b $E_{\text {merg. }}$. The number of data points, $N$, and the correlation coefficients, $\rho$, are indicated in each plot 
compute the correlation coefficients $\rho$ between $\delta R$ and $E_{\text {merg }}$ and between $\delta R$ and negative IMF $B_{z}$ as a function of MLT. The results for MLT ranges of $90 \mathrm{~min}$ (shifted by $30 \mathrm{~min}$ for each data point shown) are reported in Fig. 6a, for $\delta R$ corresponding to latitude range $60-67^{\circ}$ CGM. A delay between interplanetary and groundbased measurements was included similar to that in Sect. 2.3. No significant dependence of $\rho$ on MLT is shown in Fig. 6a. The values of $\rho$ in Fig. 6 are significantly smaller than in Fig. 3 owing to the smaller

$(60,67)$ CGM Lat. $\quad$ - E-merg. $\quad+$ Negative IMF B

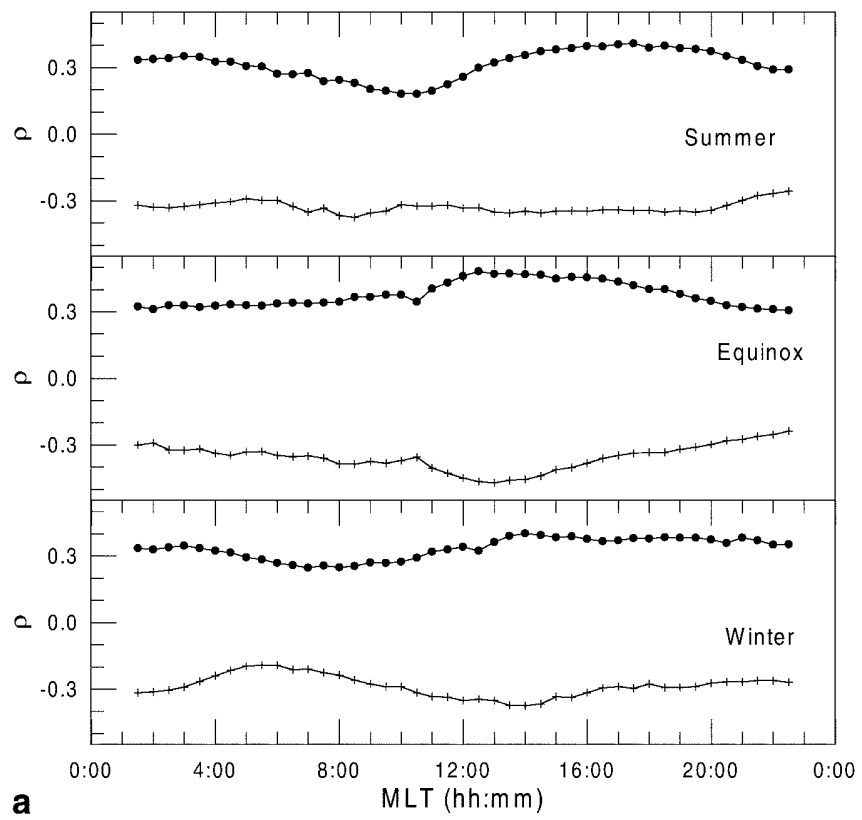

$(67,74)$ CGM Lat. - E-merg. + Negative IMF B

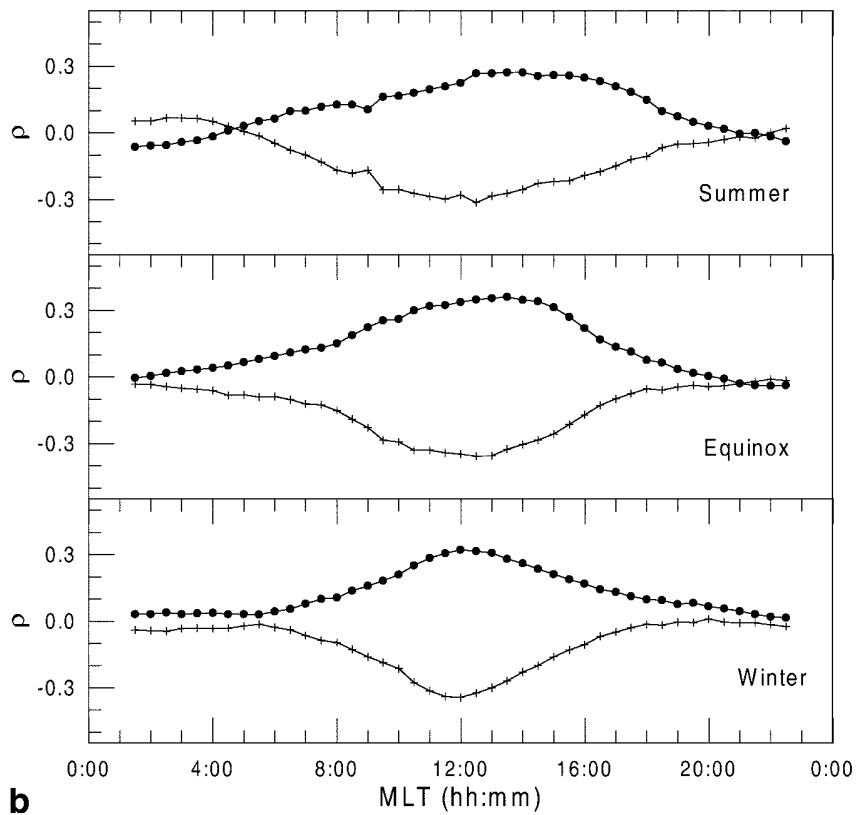

Fig. 6a, b. Correlation coefficients $\rho$ between negative IMF $B_{z}$ or $E_{\text {merg }}$ and $\delta R$ computed at 3-h MLT intervals centered at the indicated MLT; results are shown separately for latitude range a $60-67^{\circ} \mathrm{CGM}$, and b $67-74^{\circ} \mathrm{CGM}$ number of data points (and smaller variability of $B_{z}$ and $E_{\text {merg }}$ ) in each MLT interval. In Fig. 6b we show results similar to those in Fig. 6a, but in this case for the latitude range $67-74^{\circ} \mathrm{CGM}$. At these latitudes there is a large dependence of $\rho$ on MLT, and the correlations are significant only around magnetic local noon during all three seasonal periods considered. No significant correlation is found taking into account higher latitude ranges or the entire $60-90^{\circ} \mathrm{CGM}$ latitude range. In addition no significant correlations have been obtained with $N$ or with $V_{s w}$.

In order to investigate a possible relationship between the MLT dependence of $\rho$ shown in Fig. 6a, b, and a possible MLT dependence of $R$ itself, we show in Fig. 7 a histogram of the average of $R$ calculated for separate MLT intervals of $90 \mathrm{~min}$ at the two latitude ranges $60-67^{\circ} \mathrm{CGM}$ and $67-74^{\circ} \mathrm{CGM}$. A decrease in $R$ is seen around 12:00 MLT at latitudes $60-67^{\circ} \mathrm{CGM}$, and around 08:00 MLT at latitudes 67-74 ${ }^{\circ}$ CGM. In the post-midnight MLT sector the average $R$ shown in Fig. 7 is higher during the summer than during the winter, being in particular higher at latitude $60-67^{\circ}$ CGM. Viceversa, in the afternoon MLT the average $R$ is higher during the winter than during the summer, being in particular higher for the latitude range $67-74^{\circ} \mathrm{CGM}$.

\section{Radar scattering occurrence and interplanetary medium: relevant parameters}

Figure 8 shows the distributions of the values of all the interplanetary parameters during the winter months of 1997 and 1998 (an interplanetary/ground-based delay of $90 \mathrm{~min}$ was considered in agreement with previous
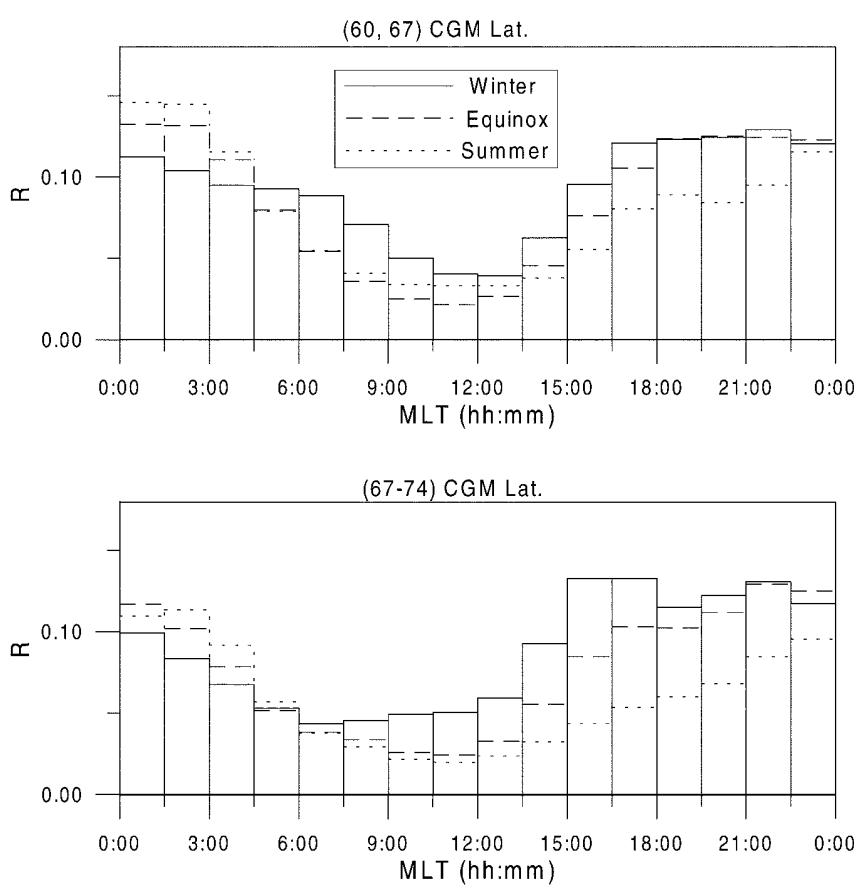

Fig. 7. Average of the scattering occurrence, $R$, at different MLT ranges and for different seasons during 1997 and 1998. Results are shown separately for latitude range $60-67^{\circ} \mathrm{CGM}$ and $67-74^{\circ} \mathrm{CGM}$ 

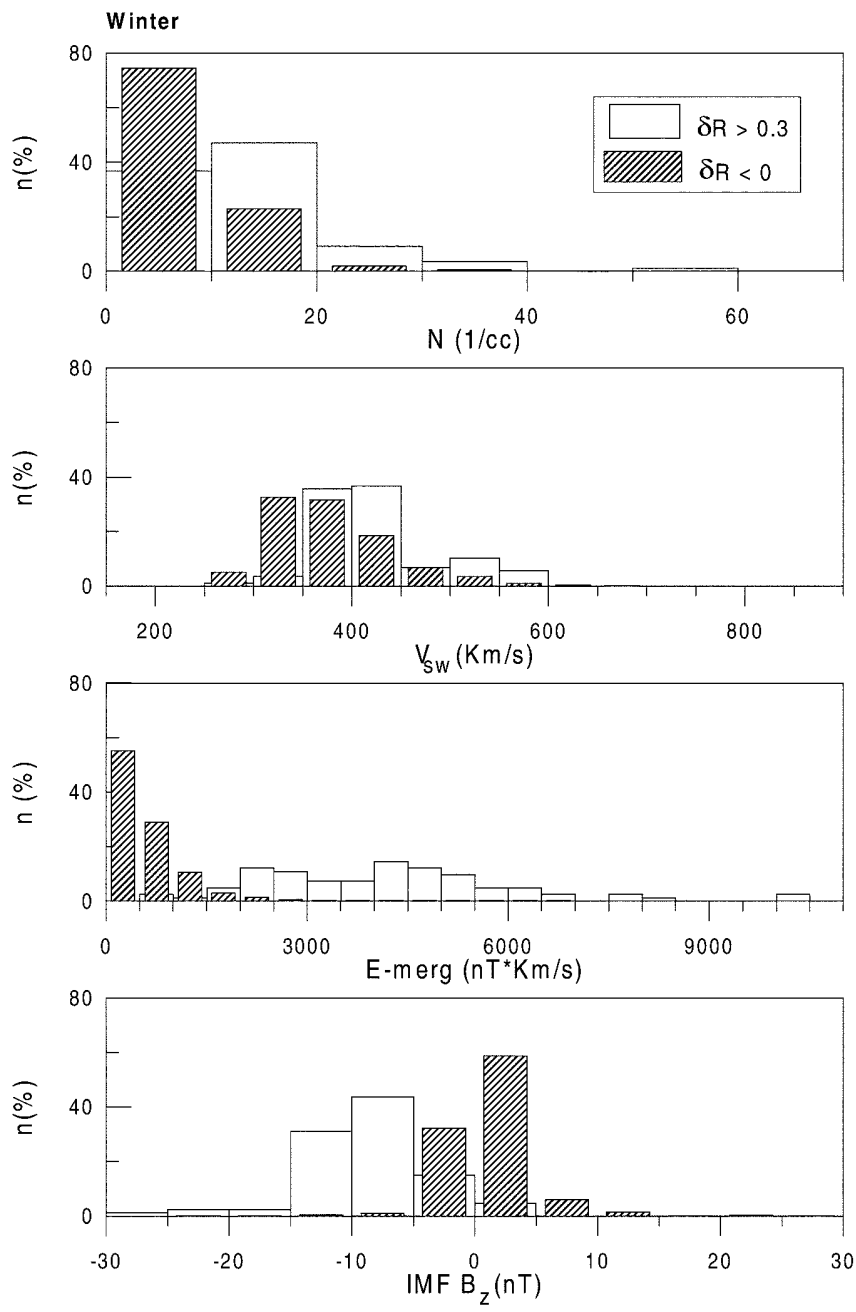

Fig. 8. Distribution of proton density $N$, solar wind speed $V_{s w}$, merging electric field $E_{\text {merg }}$ and IMF $B_{z}$ component for measurements with $\delta R<0$ and $\delta R>0.3$. Results are shown for winter periods of 1997 and 1998

findings). These histograms are shown separately for different ranges of values of $\delta R$ computed in the latitude range $60-67^{\circ}$ CGM: Fig. 8a shaded columns display results for negative values of $\delta R$, which means that the scattering occurrence is lower than its average; Fig. $8 \mathrm{~b}$ white columns display results for $\delta R>0.3$, which means that the scattering occurrence is significantly higher than its average. For $\delta R>0.3$, a clear shift towards higher values of $E_{\text {merg }}$ and negative $B_{z}$ is shown, in agreement with the correlations previously found. There is almost no overlap between the two distributions. Moreover, Fig. 8 shows that a shift of the distributions of $N$ and $V_{s w}$ toward higher values is observed also for higher values of $\delta R$, although the effect is less important. Similar results are obtained for the summer and equinox periods.

Although, as said in the previous section, the correlation between $\delta R$ and $N$, and with $V_{s w}$, are found to not be significant, the results reported in Fig. 8, however, show that these two parameters do have an effect on the scattering occurrence. This may be a signature of the effects of kinetic pressure. Calculating the correlation coefficients between $\delta R$ and the kinetic pressure $\left(N * V_{s w}^{2}\right)$, however, we note that these correlations are not statistically significant in our data set.

We note that the rate of the scattering occurrence $R$ is not a direct indication of the presence of density irregularities in the ionosphere, because this is affected also by the propagation of the sounding signal through the ionosphere and by its refraction or absorption at each specific time. In particular the rate of scattering occurrence considered here represents a lower limit for the presence of ionospheric irregularities. This does not exclude its possible use to obtain information on the ionospheric variability associated with high-latitude phenomena (e.g., Ruohoniemi and Greenwald, 1997).

\section{Discussion}

The average scattering occurrence (as shown in Figs. 4 and 7) versus magnetic latitude and MLT is found to be seasonally dependent, being generally higher during winter. This result confirms previous observations (e.g., Ruohoniemi and Greenwald, 1997; and references therein) and can be interpreted in terms of the sunlit suppression of ionopheric density gradients by more intense summer photoionization.

The results reported in Fig. 4 show that the backscatter is more common at auroral latitudes than in the polar cap, and Fig. 7 illustrates that the higher occurrence is concentrated to the afternoon and nightside sectors between about 15:00 and 02:00 MLT. We note that these observations are in agreement with previous results by Ruohoniemi and Greenwald (1997). This agreement is even more interesting if one takes into account that the definition of 'scattering occurrence' given by Ruohoniemi and Greenwald (1997) differs from ours.

We found that the time range of maximum occurrence is at about 00:00-03:00 MLT during the summer, and in the afternoon sector, from 15:00 to 18:00 MLT in winter. We can compare these results with those from the CUTLASS radars. Milan et al. (1997) found that, at an operating frequency of $10 \mathrm{MHz}$, more backscatter occurs at ranges further from the radar locations around noon local time during the winter; conversely the strongest backscatter occurs at near radar ranges and in the midnight local time sector during the summer. Given that Milan et al. (1997) considered UT, and taking into account that the average UT-MLT offset for the considered CUTLASS meridian is about $3 \mathrm{~h}$ (Baker and Wing, 1989), we find a perfect agreement with present observations. Milan et al. (1997) interpreted the seasonal differences in their scattering occurrence distribution as due to HF propagation modes in the seasonally different ionospheric propagation environments.

If we consider instead of the scattering occurrence its variation, we find that this parameter is significantly influenced by the interplanetary medium. As shown in Fig. 8, higher values of the variation of scattering can be associated with higher interplanetary proton density $N$, $V_{s w}, E_{\text {merg }}$ or more negative IMF $B_{z}$. In particular the 
results for the IMF and $E_{\text {merg }}$ are clearer, in association with a significant linear correlation related to these parameters, as discussed later.

The possible influence of $N$ and $V_{s w}$ on the variation of scattering occurrence may suggest the presence of effects related to the kinetic pressure. We note, however, that the correlation between $\delta R$ and the kinetic pressure $\left(N * V_{s w}^{2}\right)$ is not statistically significant in our data set. Moreover, considering the scattering occurrence as related to field-aligned ionospheric irregularities, it is of interest to note that the compression of the magnetosphere is found not to influence the level of observed field-aligned currents (Le et al., 2000).

If we consider the degree of correlation between $\delta R$ and $E_{\text {merg }}$ (and $B_{z}$ ), this implies an important direct influence of $E_{\text {merg }}$ (and IMF $B_{z}$ ) on the occurrence of scattering. At the same time we note that the highest correlation coefficients obtained (shown in Fig. 3) are about 0.5-0.6. This indicates that $E_{\text {merg }}$ (or IMF $B_{z}$ ) alone cannot be the only parameter which determines the scattering occurrence value at each UT. In this sense an exact study of predicting the scattering occurrence would imply the use of a more sophisticated statistics.

The results shown in Figs. 5 and 8 neverthless show the influence of the merging between the IMF and the magnetosphere on the scattering occurrence. In this sense the $E_{\text {merg }}$ is a more general indicator of this merging, including the $B_{z}$ consideration as one of its specific cases (Kan and Lee, 1979). In fact negative $B_{z}$, which corresponds to an IMF clock angle $90^{\circ}<\phi<270^{\circ}$, gives a higher contribution to the value of $E_{\text {merg }}\left(\sin ^{2}(\phi / 2)>1 / 2\right)$ than positive $B_{z}$ (for which $\left.\sin ^{2}(\phi / 2)<1 / 2\right)$. Including reconnection effects due to negative $B_{z}, E_{\text {merg }}$ is a better indicator of any possible $\mathrm{IMF} /$ magnetosphere reconnection. For example we mention that during positive $B_{z}$, for $B_{y}>>B_{z}$ and $\phi \sim 90^{\circ}\left(\sin ^{2}(\phi / 2) \sim 1 / 2\right)$ lobe reconnection is expected and taken into account in calculation of $E_{\text {merg }}$.

After these considerations, we can recall that IMF/ magnetosphere reconnection leads to an increase of geomagnetic activity (e.g. Arnoldy, 1971; Chen et al., 1997; Lu et al., 1998), so that an increase in the number of ionospheric irregularities is expected. In this sense, our results show that an increase in the variation of the scattering occurrence is associated with an increase in ionospheric irregularities driven by IMF/magnetospheric reconnection.

Although the observed seasonal dependence of the scattering occurrence itself (as shown in Figs. 4 and 7 and described already), we find no seasonal effects in the relationship between the variation of the scattering occurrence (with respect to its monthly average daily modulation) and the interplanetary medium (as seen in Figs. 3, 5 and 6). In contrast, it is found that the correlations between the variation of the scattering occurrence $\delta R$ and negative IMF $B_{z}$ or $E_{\text {merg }}$ depend on the geomagnetic latitude. In particular this correlation is statistically significant in the auroral oval but not in the polar cap.

This result cannot be simply related to the characteristics of the polar cap geophysics, which implies the presence of ionospheric irregularities and an IMF association also for the highest latitudes. In fact, periods of extensive HF scattering in the polar cap have been described in case studies (Rosenberg et al., 1993; Rodger et al., 1994; Rodger and Graham, 1996). Moreover, the significant relationship between the polar cap ionosphere and the interplanetary medium is a well known result (e.g., Troshichev et al., 1988).

The observed absence of correlation between $E_{\text {merg }}$ and the variation of scattering occurrence in the polar cap can be interpreted in terms of the differences between the HF propagation modes in the radar near or far ranges (Milan et al., 1997). Since the interplanetary conditions do not change with season while the backscatter shifts seasonally toward the radar near or far ranges (Milan et al., 1997), it is clear that internal magnetospheric/ionospheric processes affect the location of the echoes with respect to the radar field-ofview. This could explain our observation of an absence of correlation at higher latitudes. Furthermore, our result implies a more direct measurement of ionospheric irregularities in the radar at closer ranges and a more important ionospheric re-processing of the HF signal for the echoes corresponding to the far range cells. This is also in agreement with the expected characteristics of HF progation modes (Davies, 1967; Milan et al., 1997).

Considering the MLT dependence of the correlation coefficients $\rho$ between the variation of scattering occurrence $\delta R$ and $E_{\text {merg }}$ (or negative $B_{z}$ ), as shown in Fig. 6, we note that this is not associated with the MLT modulation of the scattering occurrence itself, as shown in Fig. 7. In fact, for the latitude ranges $60-67^{\circ} \mathrm{CGM}$ we found a minimum scattering occurrence around magnetic local noon, but no statistically significant MLT dependence. At latitude range $67-74^{\circ}$ CGM a minimum for the scattering occurrence is found around 08:00 MLT, while the coefficients of the considered correlations are not significant apart from around magnetic local noon. If we consider the association between HF scattering and the noon-time located cusp/ cleft (as reported by Ruohoniemi and Greenwald, 1997), we can interpret this result as evidence of an increase in the influence of $E_{\text {merg }}$ (or IMF $B_{z}$ ) on the variation of scattering occurrence in the cusp/cleft region.

\section{Summary and conclusions}

We have considered, over a two-year period, the rate of scattering occurrence from the whole array of SuperDARN radars operating in the Northern Hemisphere and found the following results.

1. In agreement with previous observations (Ruohoniemi and Greenwald, 1997; Milan et al., 1997), we found that the HF scattering occurrence depends on the MLT, the magnetic latitude and the local season.

2. Considering the variation of the scattering occurrence with respect to its monthly average daily modulation, $\delta R$, we found that the interplanetary parameter which mostly influences $\delta R$ is the merging electric field $E_{\text {merg }}$. 
A statistically significant correlation was found between $\delta R$ and $E_{\text {merg }}$ and interpreted in terms of an influence of the magnetic reconnection on HF scattering occurrence.

3. Although there exists a seasonal variation of the scattering occurrence, the relationship between $\delta R$ and $E_{\text {merg }}$ is independent of the season.

4. The magnetic latitude dependence of the correlation between the variation of scattering occurrence $\delta R$ and $E_{\text {merg }}$ has been investigated. A significant correlation was found in the auroral oval but not in the polar cap. This is explained in terms of the different nature of the echoes received from radar near or far range cells: in our observation, the internal ionospheric HF signal re-processing is considered to influence the occurrence of far range scatter more than the interplanetary medium configuration.

5. The dependence on magnetic local time of the correlation coefficients between the variation of scattering occurrence $\delta R$ and the merging electric field $E_{\text {merg }}$ has been studied. At latitudes in the range 60-67 $7^{\circ}$ CGM these coefficients are independent of MLT. Conversely, at higher latitudes (67-74 $\mathrm{CGM})$, the presence of a MLT dependence indicates a possible association with the cusp/cleft region.

Acknowledgements. Operation of the SuperDARN radars in the Northern Hemisphere, for the period of study under consideration, is supported by the National Funding Agencies of the US, Canada, the UK and France.

Topical Editor Gérard Chanteur thanks D.A. Lorentzen and K.J.F. Sedgemore-Schultken for their help in evaluating this paper.

\section{References}

Arnoldy, R. L., Signature in the interplanetary medium for substorms, J. Geophys. Res., 76, 5189, 1971.

Baker, K. B., and S. Wing, A new magnetic coordinate system for conjugate studies of high latitudes, J. Geophys. Res., 94, 9139, 1989.

Ballatore, P., J. P. Villain, M. Pick, and N. Vilmer, Some effects of the interplanetary CMEs on the rate of scattering occurrence for the SuperDARN radar array, ESA-SP 448, 965-969, 1999.

Chen, J., P. J. Cargill, and P. J. Palmadesso, Predicting solar wind structures and their geoeffectiveness, J. Geophys. Res., 102, $14701,1997$.

Davies, K., A nomenclature for oblique ionospheric soundings and ray tracing, Radio Sci., 2, 1395, 1967.
Farrugia, C. J., J. D. Scudder, M. P. Freeman, L. Janoo et al., Geoeffectiveness of three WIND magnetic clouds: a comparative study, J. Geophys. Res., 103, 17 261, 1998.

Greenwald, R. A., K. B. Baker, J. R. Dudeney, M. Pinnock, T. B. Jones, E. C. Thomas, J. P. Villain, J. C. Cerisier, C. Senior, C. Hanuise, R. D. Hunsucker, G. Sofko, J. Koehler, E. Nielsen, R. Pellinen, A. D. M. Walker, N. Sato, and H. Yamagishi, DARN/ SuperDARN: a global view of high-latitude convection, Space Sci. Rev., 71, 763, 1995.

Kan, J. R., and L. C. Lee, Energy coupling function and solar wind-magnetosphere dynamo, Geophys. Res. Lett., 6, 577, 1979.

Le, G., C. T. Russell, P. J. Chi, A. Szabo, and R. L. Lepping, The state of the magnetosphere on the day the solar wind nearly disappeared, 33rd COSPAR Scientific Assembly, 16-26 July 2000, Abstract PSW1-0044, 2000.

Lu, G., D. N. Baker, R. L. McPherron, C. J. Farrugia, D. Lummerzheim, J. M. Ruohoniemi, F. J. Rich, D. S. Evans, R. P. Lepping, M. Brittnacker, X. Li, R. Greenwald, G. Sofko, J. Villain, M. Lester, J. Thayer, T. Moretto, D. Milling, O. Troshichev, A. Zaitzev, V. Odintzov, G. Makarov, and K. Hayashi, Global energy deposition during January 1997 magnetic cloud event, J. Geophys. Res., 103, 11685 , 1998.

Milan, S. E., T. K. Yeoman, M. Lester, E. C. Thomas, and T. B. Jones, Initial backscatter occurrence statistics from the CUTLASS HF radars, Ann. Geophysicae, 15, 703, 1997.

Rodger, A. S., and A. C. Graham, Diurnal and seasonal occurrence of polar patches, Ann. Geophysicae, 14, 533, 1996.

Rodger, A. S., M. Pinnock, J. R. Dudeney, K. B. Baker and R. A. Greenwald, A new mechanism for polar patch formation, J. Geophys. Res., 99, 6425, 1994.

Rosenberg, T. J., Z. Wang, A. S. Rodger, J. R. Dudeney, and K. B. Baker, Imaging riometer and $\mathrm{HF}$ radar measurements of drifting $\mathrm{F}$ region electron density structures in the polar cap, J. Geophys. Res., 98, 7757, 1993.

Ruohoniemi, J. M., and R. A. Greenwald, Rates of scattering occurrence in routine HF radar observations during solar cycle maximum, Radio Sci., 32, 1051, 1997.

Ruohoniemi, J. M., R. A. Greenwald, K. B. Baker, J. P. Villain, and M. A. McCready, Drift motion of small-scale irregularities in the high-latitude F region: an experimental comparison with plasma drift motion, J. Geophys. Res., 92, 4553, 1987.

Russell, C. T., G. Le, P. Chi, X.-W. Zhou, J.-H. Shue, S. M. Petrinec, P. Song, F. R. Fenrich, and J. G. Luhmann, The extreme compression of the magnetosphere on the May 41998 as observed by the POLAR spacecraft, Adv. Space Res., 25, 1369, 2000.

Troshichev, O. A., V. G. Andrezen, S. Vennerstrøm, and E. FriisChristensen, Magnetic activity in the polar cap - a new index, Planet. Space Sci., 36, 1988.

Villain, J. P., R. A. Greenwald, and J. F. Vickrey, HF ray tracing at high latitudes using measured meridional electron density distribution, Radio Sci., 19, 359, 1984. 